\title{
Comparison of the Pharmacokinetics of RIPK1 Inhibitor GSK2982772 in Healthy Western and Japanese Subjects
}

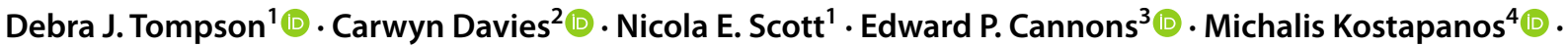

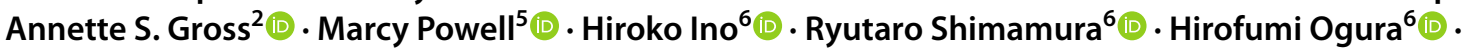

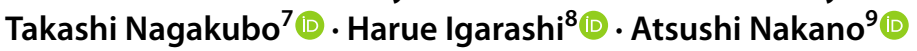

Published online: 9 November 2020

(c) The Author(s) 2020

\begin{abstract}
Background and Objectives GSK2982772 is an oral small-molecule RIPK1 inhibitor with potential therapeutic efficacy in immune-mediated inflammatory diseases (IMIDs). An inter-ethnic comparison of GSK2982772 pharmacokinetics was conducted based on data from Western (Study 1) and Japanese subjects (Study 2).

Methods Both studies were single-centre, randomised, double-blind, placebo-controlled studies with objectives to assess the safety and characterise the pharmacokinetics of GSK2982772. Western subjects in Study 1 (NCT03305419), Part A $(N=15)$, were randomly assigned to receive $120 \mathrm{mg}$ three times daily (TID), $240 \mathrm{mg}$ TID, or $360 \mathrm{mg}$ twice daily (BID) doses of GSK2982772, or placebo (TID or BID) for 1 day. Part B subjects $(N=47)$ received GSK2982772 $120 \mathrm{mg}$ TID, $240 \mathrm{mg}$ TID, or placebo TID for 14 days. Japanese subjects in Study $2(N=13)$ (NCT03590613) were randomly assigned to receive TID doses of GSK2982772 60, 120, $240 \mathrm{mg}$ TID or placebo TID for 1 day.

Results GSK2982772 was well tolerated and adverse events were generally mild. Maximum observed plasma drug concentration $\left(C_{\max }\right)$, time to reach $C_{\max }\left(T_{\max }\right)$, area under the plasma drug concentration versus time curve after the first GSK2982772 dose $\left(\mathrm{AUC}_{(0-7)}\right)$ of 120 and $240 \mathrm{mg}$, and $\left(\mathrm{AUC}_{(0-24)}\right)$ values for the 120 and $240 \mathrm{mg}$ TID doses over a single day were similar in Japanese and Western subjects.

Conclusions The pharmacokinetics and tolerability of GSK2982772 were similar between Western and Japanese subjects, justifying inclusion of Japanese subjects in future global clinical studies to assess the therapeutic potential of RIPK1 inhibition for the treatment of IMIDs.

Clinical Trials: NCT03305419 and NCT03590613 available from http://www.clinicaltrials.gov.
\end{abstract}

\section{Key Points}

TID and BID dosing up to $720 \mathrm{mg}$ daily of GSK2982772 was well tolerated in Western and Japanese subjects.

Similar pharmacokinetics and tolerability of GSK2982772 between Western and Japanese subjects support inclusion of Japanese subjects in future global studies.

Electronic supplementary material The online version of this article (https://doi.org/10.1007/s13318-020-00652-2) contains supplementary material, which is available to authorized users.

Debra J. Tompson

debra.j.tompson@gsk.com

Extended author information available on the last page of the article 


\section{Introduction}

GSK2982772 binds selectively to an allosteric pocket of the receptor-interacting protein kinase 1 (RIPK1) domain to inhibit RIPK1-mediated programmed cell death pathways and pro-inflammatory cytokine production [1]. Inhibitors of RIPK1 can modulate necroptosis, a form of programmed cell death that may play a role in the pathogenesis of immune-mediated inflammatory diseases (IMIDs), and thus GSK2982772 has emerged as a potential treatment for IMIDs such as psoriasis, rheumatoid arthritis, and inflammatory bowel disease [1-3].

Following oral administration of GSK2982772 as powder in a capsule or a tablet, GSK2982772 was rapidly absorbed with a median time to maximum observed plasma concentration $\left(C_{\text {max }}\right.$ ) of $1.5-2.5$ h post-dose. Approximate linear kinetics were displayed over the dose range of $0.1-120 \mathrm{mg}$ and food had no significant impact on the rate or extent of absorption [1]. The majority of systemic exposure to GSK2982772 was associated with a half-life of $2-3 \mathrm{~h}$. Consequently, initial experimental medicine studies conducted with GSK2982772 for plaque-type psoriasis, rheumatoid arthritis, and ulcerative colitis used twice daily (BID) and three time a day (TID) dosage regimens [4].

Following single-day and repeat dosing (120 mg BID) over 14 days, the principal components in blood and plasma were unchanged GSK2982772 and an $N$-glucuronide metabolite (M8; GSK3562183) [1]. A minor desmethyl metabolite (M9; GSK2997852) was also present in plasma. In vitro studies indicate that UGT1A9 was the predominant enzyme catalysing the formation of M8 with minor contributions from UGT1A1 and UGT1A7.

This current analysis compares the pharmacokinetics and safety of GSK2982772 in healthy Western (predominantly White/European) subjects (Study 1) and Japanese subjects (Study 2). The main objectives of the Western study were to determine the pharmacokinetics and safety of GSK2982772 at higher single-day (120 mg TID, $240 \mathrm{mg}$ TID, and $360 \mathrm{mg}$ BID) and 14 day repeat doses (120 and $240 \mathrm{mg}$ TID) than studied previously in initial experimental medicine studies (60 mg TID and $120 \mathrm{mg}$ BID). The objective of the Japanese study was to determine the safety, tolerability, and pharmacokinetics of GSK2982772 at doses up to $240 \mathrm{mg}$ TID for a single day to support the inclusion of Japanese subjects in global phase $2 b$ clinical trials and to explore the potential for inter-ethnic differences in the pharmacokinetics of GSK2982772.

Although the overall designs of the two studies were not the same, both studies included a single-day administration of $120 \mathrm{mg}$ TID and $240 \mathrm{mg}$ TID, which allowed for comparisons of pharmacokinetics in healthy Japanese and Western subjects. GSK2982772 was administered as tablets in the Japanese study and as capsules in the Western study. Both formulations of GSK2982772 have a similar rate and extent of absorption [1]. In vitro human hepatocyte studies with GSK2982772 indicated a moderate potential for induction of cytochrome P450 enzymes at clinical doses of $240 \mathrm{mg}$ TID. Therefore, in Study 1 the plasma $4 \beta$-hydroxycholesterol-to-cholesterol ratio was measured as a biomarker of CYP3A4 activity [5].

\section{Methods}

\subsection{Study Design}

Study 1 (NCT03305419; initiated October 11, 2017, and completed October 15, 2018, at a single centre in the UK) was a randomised, double-blind (excluding the site pharmacist), placebo-controlled study divided into two parts (Fig. 1). In Part A, subjects (cohort 1) were randomly assigned in a three-way crossover design to receive ascending doses of GSK2982772 $120 \mathrm{mg}$ TID, $240 \mathrm{mg}$ TID, or $360 \mathrm{mg}$ BID for 1 day. In Part B, subjects received repeat doses of GSK2982772 $120 \mathrm{mg}$ TID in cohorts 2 and 3 and $240 \mathrm{mg}$ TID in cohort 4 for 14 days in a sequential group design.

In cohorts 1 and 2, subjects were randomised 3:1 to receive either GSK2982772 or placebo. However, due to an unexpectedly high number of asymptomatic arrhythmias, the study was paused after cohort 2 . The ratio of subjects on active treatment versus placebo changed in cohorts 3 and 4 to 9:5 to more closely monitor the effects of GSK2982772 relative to placebo, including the $120 \mathrm{mg}$ TID dose. A fifth cohort was planned (360 mg BID) but was not initiated as one subject at the $360 \mathrm{mg}$ BID dose level in cohort 1 exceeded the predefined $C_{\max }$ stopping criteria.

For TID dosing in both parts of Study 1, GSK2982772 or placebo was administered at 0,7 , and $14 \mathrm{~h}$, with the first dose administered following an overnight fast. The $360 \mathrm{mg}$ BID doses in Part A were administered at 0 and $12 \mathrm{~h}$. The impact of food on steady-state GSK2982772 pharmacokinetic parameters was evaluated during the repeat dose phase in Part B. GSK2982772 was administered 30 min after a standard breakfast on Day 9 or after a high-fat breakfast on Day 11, and in the fasted state on Day 14, with the content of each breakfast aligned to US FDA recommendations [6]. On fasting days, breakfast was served approximately $2 \mathrm{~h}$ after dosing, and on all study days, lunch and dinner were served 2-3 h prior to doses 2 and 3, respectively.

Study 2 (NCT03590613; initiated July 19, 2018, and completed September 26, 2018) was a single-centre, randomised, double-blind, placebo-controlled study conducted in Japan (Fig. 1). Subjects were randomly assigned 


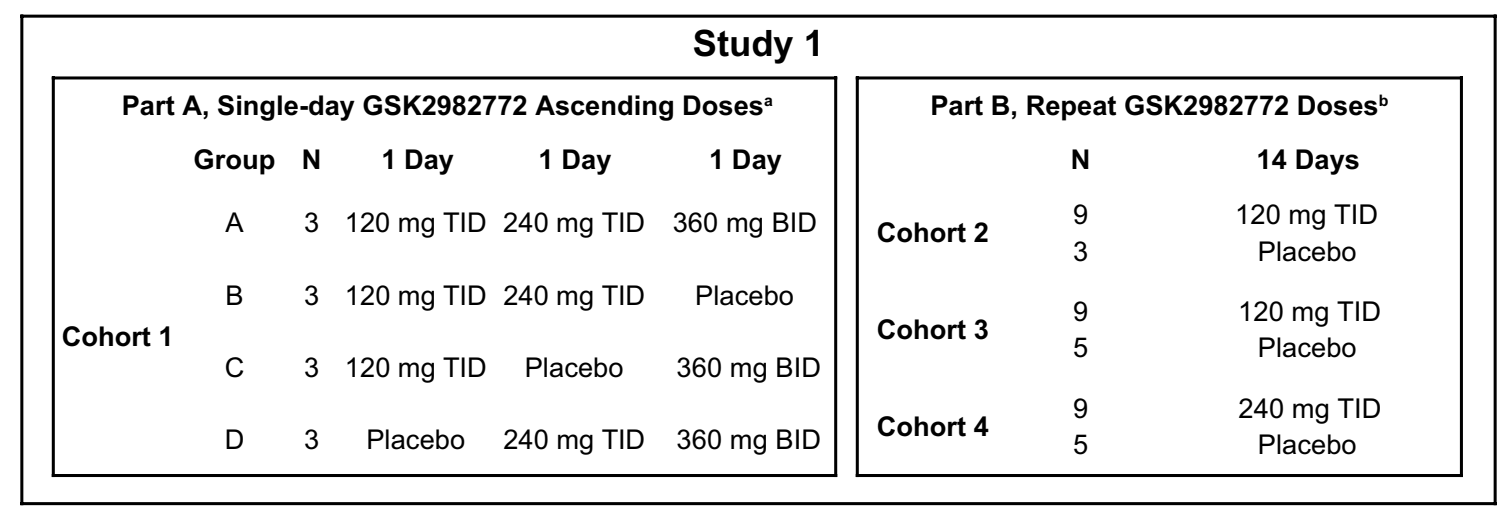

\begin{tabular}{|cccccc|}
\hline \multicolumn{6}{c|}{ Study 2 Single Daya } \\
Group & N & 1 Day & 1 Day & 1 Day & 1 Day \\
A & 3 & Placebo TID & $60 \mathrm{mg}$ TID & 120 mg TID & $240 \mathrm{mg}$ TID \\
B & 3 & $60 \mathrm{mg}$ TID & Placebo TID & $120 \mathrm{mg}$ TID & $240 \mathrm{mg}$ TID \\
C & 3 & $60 \mathrm{mg}$ TID & $120 \mathrm{mg}$ TID & Placebo TID & $240 \mathrm{mg}$ TID \\
D & 3 & $60 \mathrm{mg}$ TID & $120 \mathrm{mg}$ TID & $240 \mathrm{mg}$ TID & Placebo TID \\
\hline
\end{tabular}

Fig. 1 Design of the Western (Study 1) and Japanese (Study 2) studies. A fifth cohort (360 mg BID) was planned in Study 1 but was not conducted because one subject in cohort 1 exceeded the $C_{\max }$ stopping criteria at the $360 \mathrm{mg}$ BID dose level. ${ }^{\mathrm{a}} \mathrm{A}$ 7-day washout period

1:1:1:1 in a four-way crossover design to receive ascending doses of GSK2982772 $60 \mathrm{mg}$ TID, $120 \mathrm{mg}$ TID, $240 \mathrm{mg}$ TID, and placebo TID for 1 day, using the same TID dosing interval as Study 1.

In Study 1, GSK2982772 was administered in capsule formulation. In Study 2, GSK2982772 was administered as a tablet formulation [1]. In both studies, placebo was matched in appearance to the active study drug. For each study, approval was obtained from the ethics committee of the clinical trial unit and each was conducted according to the recommendations of Good Clinical Practice and the Declaration of Helsinki (2013). Written informed consent was obtained from each subject prior to any study-related activities.

\subsection{Subjects}

Healthy male and female subjects aged $18-65$ years, $\geq 50 \mathrm{~kg}$, and body mass index (BMI) $19-30 \mathrm{~kg} / \mathrm{m}^{2}$ were eligible for Study 1. The inclusion criteria for Study 2 included healthy male Japanese subjects, aged $20-64$ years, $\geq 50 \mathrm{~kg}$, and BMI $18.5-24.9 \mathrm{~kg} / \mathrm{m}^{2}$. Full eligibility criteria are included in Supplemental Table 1. was required between each single-day dosing period in Study 1, Part

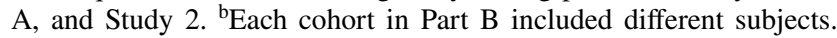
$B I D$ twice daily, $C_{\max }$ maximum blood/plasma concentration, TID three times daily

\subsection{Endpoints}

Primary endpoints of both studies were the safety and tolerability of GSK2982772 (see Sect. 2.4).

The secondary endpoints for both studies were GSK2982772 pharmacokinetic parameters. In Study 1, Part B, the impact of repeat dosing of GSK2982772 for 14 days and the impact of co-administration of GSK2982772 with a standard and high-fat meal were determined.

The derived pharmacokinetic parameters were maximum observed plasma concentration $\left(C_{\max }\right)$ and time to $C_{\max }\left(T_{\max }\right)$ following each dose, area under the plasma drug concentration versus time curve over $24 \mathrm{~h}\left(\mathrm{AUC}_{(0-24)}\right)$ and over each dose interval $\left(\mathrm{AUC}_{(0-7)}, \mathrm{AUC}_{(7-14)}\right.$, and $\mathrm{AUC}_{(14-24)}$ for TID dosing and $\mathrm{AUC}_{(0-12)}$ and $\mathrm{AUC}_{(12-24)}$ for BID dosing). Observed trough plasma drug concentrations were also assessed in both studies. The $\left(t_{1 / 2}\right)$ following the third dose was assessed in Japanese participants only.

Study 1 explored more than two main endpoints of safety and pharmacokinetics. In Study 1, Part B, the potential effect of repeat doses of GSK2982772 on CYP3A4 enzyme activity was assessed by comparing the ratio of plasma $4 \beta$-hydroxycholesterol to cholesterol on Day 15 versus Day 1. 


\subsection{Safety Assessments and Tolerability}

Safety and tolerability assessments included monitoring of adverse events (AEs), clinical laboratory values, vital signs (heart rate, blood pressure, and body temperature), electrocardiogram, and physical examination. AEs and serious AEs (SAEs) were collected from the start of study treatment through the follow-up visit.

Assessment of the AEs/SAEs included evaluation of intensity as 'mild', 'moderate', or 'severe' as assessed by the investigator. All AEs/SAEs were followed by the investigator until resolution/stabilisation. AE and SAE reporting was performed according to the GCP Guidelines and the National regulatory authorities' guidelines of the countries where the two studies were performed.

\subsection{Blood Sampling}

For the TID dosing regimen, blood samples were collected pre-dose, $20 \mathrm{~min}, 40 \mathrm{~min}, 1,1.5,2,3$, and $5 \mathrm{~h}$ following each dose of the single-day study period. For BID dosing, samples were collected pre-dose, $20 \mathrm{~min}, 40 \mathrm{~min}, 1,1.5,2$, $3,4,6$, and $10 \mathrm{~h}$ after each dose. For TID dosing, pre-dose samples were taken just prior to the 7- and 14-h doses. For BID dosing, a pre-dose sample was taken just prior to the 12-h dose. The pharmacokinetic blood sampling schedule for Part B of Study 1 is shown in Supplemental Table 2. On Days 2, 3, 4, 5, and 7, only pre-dose samples were obtained. On Day 14, blood sampling over $24 \mathrm{~h}$ was the same as the schedule for Part A. At all time points, $2 \mathrm{ml}$ of whole blood samples was collected into K3 EDTA tubes.

\subsection{Drug Assay}

GSK2982772 plasma concentrations were measured for Study 1 at GSK, Ware, UK, and for Study 2 the assay was performed by Covance Laboratories (Harrogate, UK). For Study 1, the lower (LLQ) and higher (HLQ) limits of quantification for GSK2982772 were $5 \mathrm{ng} / \mathrm{ml}$ and $5000 \mathrm{ng} / \mathrm{ml}$ using a $50 \mu \mathrm{l}$ aliquot of human plasma. For Study 2, there was a low (LLQ and HLQ of $0.2 \mathrm{ng} / \mathrm{ml}$ and $200 \mathrm{ng} / \mathrm{ml}$ ) and a high (LLQ and HLQ of $1 \mathrm{ng} / \mathrm{ml}$ and $1000 \mathrm{ng} / \mathrm{ml}$ ) concentration range method using a $30 \mu \mathrm{l}$ aliquot (low) and a $25 \mu \mathrm{l}$ aliquot (high) of human plasma.

For both assays, human plasma samples were analysed for GSK2982772 concentrations using a validated method (according to GSK standard operating procedures and bioanalytical method validation guidelines from the FDA and EMA) [7, 8] based on protein precipitation, followed by HPLC/MS/MS analysis equipped with API5500 (GSK) and API4000 (Covance) manufactured by Sciex, Framingham, MA, USA. GSK2982772 was extracted from human plasma via protein precipitation with acetonitrile containing an isotopically labelled internal standard, $\left[{ }^{2} \mathrm{H}_{3}\right.$ $\left.{ }^{13} \mathrm{C}\right]$-GSK2982772.

Chromatography was completed on a C18 BEH column $(50 \times 2.1 \mathrm{~mm}, 1.7 \mu \mathrm{M}$, Waters, Milford, MA, USA) under linear gradient conditions; the mobile phases were (A) water containing $0.1 \%(\mathrm{v} / \mathrm{v})$ formic acid and (B) acetonitrile. Quantitation was completed using multiple reaction monitoring (MRM) in positive mode producing transitions for GSK2982772 ( $\mathrm{m} / \mathrm{z}$ 378/204 (GSK) and $\mathrm{m} / \mathrm{z}$ 378/255 (Covance) and $\left[{ }^{2} \mathrm{H}_{3}{ }^{13} \mathrm{C}\right]-G S K 2982772(\mathrm{~m} / \mathrm{z} 382 / 255)$. The intra- and inter-assay bias and precision were $<15 \%$.

Quality control (QC) samples containing GSK2982772 at three different concentrations for Study 1 and four different concentrations for Study 2 were stored with study samples and analysed with each batch of samples against calibration standards. No more than one-third of the QC results were to deviate from the nominal concentration by more than $15 \%$, and at least $50 \%$ of the results from each QC concentration should be within $15 \%$ of nominal.

\subsection{Pharmacokinetic Analysis}

Pharmacokinetic parameters were determined from plasma GSK2982772 concentration-time data using a standard noncompartmental analysis with Phoenix WinNonlin version 6.3 [Certara USA, Inc., Princeton, NJ, USA]. Calculations were based on the actual sampling times recorded during the study.

The following pharmacokinetic parameters were determined from the plasma concentration-time data: maximum observed concentration $\left(C_{\max }\right)$ following each dose, time to maximum observed plasma drug concentration $\left(T_{\max }\right)$ following each dose, area under the curve (AUC) from time 0 to $24 \mathrm{~h}\left(\mathrm{AUC}_{(0-24)}\right)$ and over each dose interval $\left(\mathrm{AUC}_{(0-7)}\right.$, $\mathrm{AUC}_{(7-14)}$ and $\left.\mathrm{AUC}_{(14-24)}\right)$, and terminal phase half-life $\left(t_{1 / 2}\right)$ following the third dose (Japanese study only). AUC was calculated using the linear and logarithmic trapezoidal methods for increasing and decreasing concentrations, respectively. Terminal phase half-life was calculated as $\ln 2 /$ terminal elimination rate constant $(\lambda z)$.

\subsection{Biomarker Assessment}

Plasma concentrations of $4 \beta$-hydroxycholesterol and cholesterol were measured by Covance Laboratories (Harrogate, UK). Human plasma samples were analysed for cholesterol using a validated analytical method based on saponification and liquid-liquid extraction, followed by LC-MS/MS analysis. LLQ was $500 \mu \mathrm{g} / \mathrm{ml}$ using a $50 \mu \mathrm{l}$ aliquot of diluted human plasma with a HLQ of $5000 \mu \mathrm{g} / \mathrm{ml}$. Human plasma samples were analysed for 4B-hydroxycholesterol using a validated analytical method based on liquid-liquid extraction and derivatization followed by LC-MS/MS analysis. 
The LLQ was $4 \mathrm{ng} / \mathrm{ml}$ using a $25 \mu \mathrm{l}$ aliquot of human plasma with a HLQ of $400 \mathrm{ng} / \mathrm{ml}$.

\subsection{Statistical Analysis}

Safety and tolerability were summarised for all subjects who received at least one dose of study drug (safety population). Pharmacokinetic analyses were based on the pharmacokinetic population, which included all subjects with a pharmacokinetic sample analysed. Plasma concentrations at each time point were summarised using mean and standard deviation (SD) and presented in graphical form. AUC, $C_{\max }$, and $t_{1 / 2}$ were summarised using geometric mean and between-subject coefficient of variation $(\% \mathrm{CVb})$ around the geometric mean pharmacokinetic values. $T_{\max }$ was summarised using median and range. Post-hoc analyses included calculation of ratios for geometric mean $\mathrm{AUC}$ and $C_{\max }$ (Japan:Western) and graphical comparisons of AUC and $C_{\max }$ values using box (25th-75th percentiles with median) and whisker (10th-90th percentiles) plots at each dose level.

\section{Results}

\subsection{Number of Subjects and Demographics}

In Study 1, Part A, 15 healthy subjects were randomised and $12(80 \%)$ completed the study (Fig. 2). Two subjects (13\%) were withdrawn because of nonserious AEs (120 mg TID, hypertension; $240 \mathrm{mg}$ TID, hematochezia and feces soft). At the discretion of the Principal Investigator (PI), one subject (7\%) was withdrawn after the second treatment period as their $C_{\max }$ values at both doses were higher than other subjects and for the next single-day $360 \mathrm{mg}$ BID dose were predicted to exceed the pharmacokinetic stopping criteria of $12.3 \mu \mathrm{g} / \mathrm{dl}$.

In Part B, 47 subjects were randomised, with 29 (62\%) completing the study (Fig. 2). Seven subjects (15\%) withdrew because of AEs: one subject receiving placebo (ventricular tachycardia), two subjects in the $120 \mathrm{mg}$ TID group (ventricular tachycardia, hordeolum), and four subjects in the $240 \mathrm{mg}$ TID group (anxiety, influenza-like illness, mood altered, nightmare, pollakiuria, polyuria, and tachycardia). Additionally, 11 (23\%) subjects were withdrawn at the discretion of the PI after cardiac events were observed in $6 / 25$ dosed subjects in Part A, cohort 1, and Part B, cohort 2. Consequently, all ten subjects in cohort 2 who had been dosed up to that point were withdrawn.

In Study 2, 13 healthy subjects were enrolled and 12 completed the study. Drug dosing in Period 2 was postponed for one subject because of elevated aspartate aminotransferase (AST) and alanine transaminase (ALT) on Day 1 of treatment Period 2. The revised study schedule was no longer suitable, so the subject was withdrawn at the discretion of the PI.

Subject demographics and baseline characteristics for both studies are shown in Table 1. For Study 2, subjects were, on average, younger (26 years) and weighed less
Fig. 2 Number of subjects. a Western subjects, b Japanese subjects. Once an allocation number was assigned to a subject, it was not reassigned to any other subject. When a subject prematurely discontinued the study, replacement subjects were recruited and assigned to the same sequence as the replaced subject

\section{A Study 1 Western Subjects}

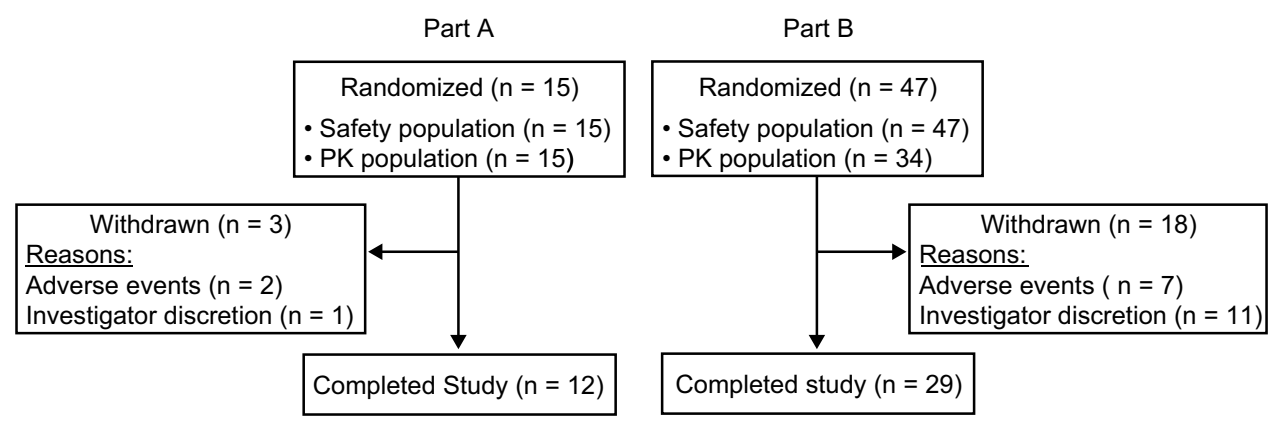

B Study 2 Japanese Subjects

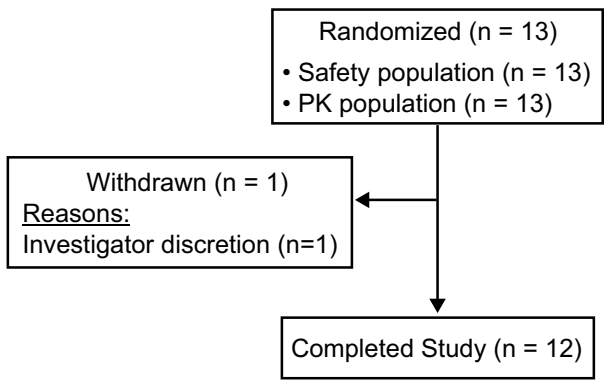


Table 1 Subject demographics and baseline characteristics (safety population)

\begin{tabular}{|c|c|c|c|c|c|c|}
\hline \multirow[t]{3}{*}{ Demographics } & \multicolumn{5}{|l|}{ Study 1} & \multirow[t]{3}{*}{ Study $2(N=13)$} \\
\hline & \multirow[t]{2}{*}{ Part A $(N=15)$} & \multicolumn{4}{|l|}{ Part B } & \\
\hline & & Placebo $(N=14)$ & $120 \mathrm{mg} \operatorname{TID}(N=20)$ & $240 \mathrm{mg}$ TID $(N=13)$ & Total $(N=47)$ & \\
\hline Age (years), [mean (SD)] & $45.0(11.6)$ & $39.1(9.7)$ & $43.3(10.9)$ & $38.6(11.0)$ & $40.7(10.6)$ & $26.2(7.3)$ \\
\hline \multicolumn{7}{|l|}{$\operatorname{Sex}[n(\%)]$} \\
\hline Female & $5(33)$ & 0 & 0 & 0 & 0 & 0 \\
\hline Male & $10(67)$ & $14(100)$ & $20(100)$ & $13(100)$ & $47(100)$ & $13(100)$ \\
\hline Height (cm), [mean (SD)] & $173.7(9.0)$ & $176.6(6.1)$ & $178.3(7.6)$ & $175.9(5.3)$ & $177.1(6.5)$ & $173.0(4.0)$ \\
\hline Weight (kg), [mean (SD)] & $78.1(14.8)$ & $78.0(8.2)$ & $85.1(12.0)$ & $76.2(12.1)$ & - & $64.0(6.3)$ \\
\hline \multicolumn{7}{|l|}{ Ancestry $[n(\%)]$} \\
\hline White/Caucasian/European & $13(87)$ & $12(86)$ & $19(95)$ & $10(77)$ & $41(87)$ & 0 \\
\hline African American/African & $2(13)$ & 0 & $1(5)$ & $3(23)$ & $4(9)$ & 0 \\
\hline Asian-Japanese & 0 & $1(7)$ & 0 & 0 & $1(2)$ & $13(100)$ \\
\hline Asian-East Asian & 0 & $1(7)$ & 0 & 0 & $1(2)$ & 0 \\
\hline
\end{tabular}

$S D$ standard deviation, $T I D$ three times daily

$(64.0 \mathrm{~kg})$ than subjects from Study 1 (Part A: 45 years, $78.1 \mathrm{~kg}$; Part B: 40 years, $81.0 \mathrm{~kg}$ ). Most subjects in Study 1 were of White/European ancestry $(87 \%)$, while all subjects in Study 2 were Japanese and residing in Japan. Study 1, Part A, included five female subjects (33\%), while all subjects in Part B and Study 2 were male.

\subsection{Adverse Events}

A summary of AEs that occurred in $>1$ subject is presented in Supplemental Table 3. Twenty subjects (56\%) reported AEs in Study 1, Part A, and 38 (81\%) reported AEs in Part B. Across both parts of Study 1, the most frequently reported AE was contact dermatitis [Part A, 9 subjects (25\%); Part B, $8(17 \%)$ ], which was attributed to the electrocardiogram and telemetry electrodes. The incidence of AEs was highest in the GSK2982772 360 mg BID single-day dosing group [7 (78\%)], followed by the $120 \mathrm{mg}$ TID group [6 (67\%)], then the $240 \mathrm{mg}$ TID group [4 subjects (44\%)] and the placebo group [3 subjects (33\%)]. In Part B, the incidence of AEs was highest in the $240 \mathrm{mg}$ TID repeat dose group [12 (92\%)], followed by the $120 \mathrm{mg}$ TID group [17 (85\%)] and the placebo group [9(64\%)].

Adverse events of moderate intensity reported in Study 1 are presented in Supplemental Table 4. In Part B, there was one serious AE reported in a male subject who received placebo and experienced a mild grade 1 cerebrovascular accident on Day 15, 1 day after the last dose.

In Part A, all study drug-related AEs [4 subjects $(44 \%)]$ were of mild intensity. In Part B, one subject in the GSK2982772 $120 \mathrm{mg}$ TID group developed an asymptomatic, non-serious elevated ALT [7.5 $\times$ the upper limit of normal (ULN)] and AST ( $4.5 \times \mathrm{ULN})$ on Day 15,1 day after the last dose. ALT and AST levels returned to normal within 4 weeks. The elevated ALT AE was severe in intensity and considered related to the study drug due to the absence of another plausible explanation. All other study drug-related AEs in Part B were mild [2 (10\%)] or moderate [2 subjects (15\%)]

In Parts A and B, six subjects experienced arrhythmia events that led to a study pause. Two subjects taking placebo had atrial tachycardia and four subjects receiving GSK2982772 had the appearance of non-sustained ventricular tachycardia (NSVT). All subjects participating in the 14-day repeat dose cohorts had extended periods of telemetry monitoring on Days 1-4, Day 14, and Day 15 after completion of dosing. While the observed cardiac events were asymptomatic and not considered medically serious, the PI considered the events possibly study drug-related. The incidence seemed unexpectedly high in this healthy population, and therefore the trial was halted as a precautionary measure and further cardiac monitoring implemented. All subjects with an arrhythmia event had an echocardiogram and follow-up with a 48-h Holter monitor well beyond five half-lives of GSK2982772. Following an unblinded evaluation of arrhythmia events, the GSK Sponsor Team and the Cardiovascular Safety Panel concluded that the asymptomatic telemetry abnormalities were unlikely to be related to GSK2982772 exposure. The AE profile and safety assessments (vital signs, laboratory values) of all subjects with an arrhythmia were reviewed, with no contributory findings apparent. Substantial protocol amendments were implemented (baseline 24-h telemetry monitoring and additional placebo subjects), and the study was restarted by repeating the repeat dose $120 \mathrm{mg}$ TID cohort. After the study restarted, there was one additional NSVT event in a placebo subject. 
In Study 2, one subject in the $60 \mathrm{mg}$ TID group (8\%) experienced a mild ALT increase (84 IU/l; normal range 5-45 IU/l) and a mild AST increase (100 IU/l; normal range 10-40 IU/1) during the first 1-day dosing period that was reported to be study drug-related (Supplemental Table 5). No other AEs were reported in Study 2.

\subsection{Pharmacokinetics}

\subsubsection{Western Subjects}

In Study 1, Part A, the shape of the GSK2982772 plasma concentration-time profiles was similar across the $120 \mathrm{mg}$ and $240 \mathrm{mg}$ TID dose groups (Fig. 3). $C_{\max }$ tended to be higher following the second dose for all BID and TID dosing regimens in Part A (Table 2). For the TID regimens, the absorption rate was slower following the third dose, with a prolonged $T_{\max }$ and lower $C_{\max }$ relative to the first and second doses. $C_{\max }$ and $\mathrm{AUC}_{(0-24)}$ values increased approximately dose proportionately between the $120 \mathrm{mg}$ and $240 \mathrm{mg}$ TID doses, and $\mathrm{AUC}_{(0-24)}$ was similar for the $240 \mathrm{mg}$ TID and $360 \mathrm{mg}$ BID regimens (total dose $720 \mathrm{mg}$ ). GSK2982772 pharmacokinetic parameters $\left(\mathrm{AUC}_{(0-24)}, C_{\max }\right.$, $\left.\mathrm{AUC}_{(0-7)}\right)$ in the limited number of female participants in Study 1 were similar to those observed in healthy males (data not shown).

One subject in the $360 \mathrm{mg}$ BID group had a $C_{\max }$ value of $16.0 \mu \mathrm{g} / \mathrm{ml}$, which exceeded the $C_{\max }$ stopping criteria $(12.3 \mu \mathrm{g} / \mathrm{ml})$. Therefore, no further doses of $360 \mathrm{mg}$ BID were administered in Part B.

In Study 1, Part B, the shape of the GSK2982772 plasma concentration-time profiles and the derived pharmacokinetic parameters were similar to those observed in Part A for the $120 \mathrm{mg}$ and $240 \mathrm{mg}$ TID regimens. $C_{\max }$ and $\mathrm{AUC}_{(0-24)}$

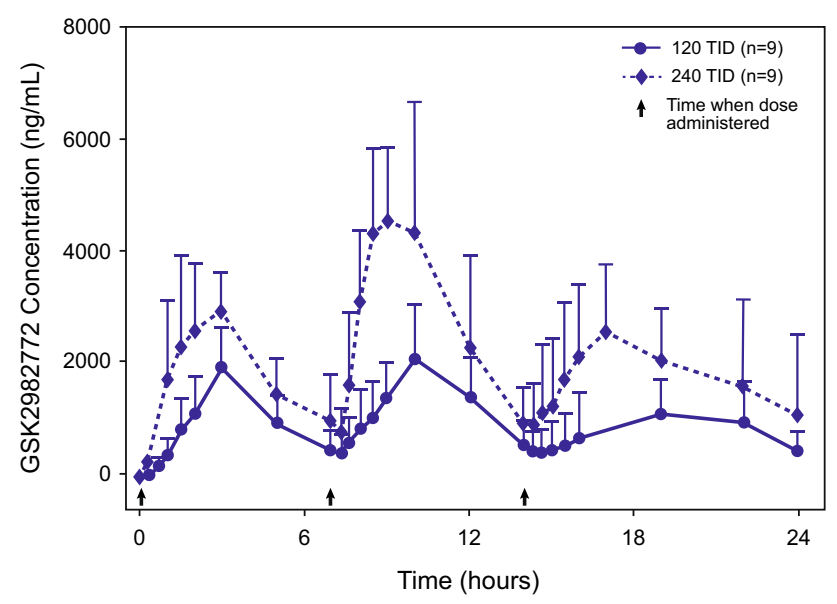

Fig. 3 Plasma GSK2982772 concentration-time profiles (arithmetic mean \pm SD) for $120 \mathrm{mg}$ TID and $240 \mathrm{mg}$ TID in Western subjects. $S D$ standard deviation, TID three times daily values increased approximately linearly between $120 \mathrm{mg}$ TID and $240 \mathrm{mg}$ TID. There was no evidence of accumulation of GSK2982772 following repeat doses of $120 \mathrm{mg}$ TID or $240 \mathrm{mg}$ TID based on no appreciable increase in $\mathrm{AUC}_{(0-7)}$ on Day 14 versus Day 1. GSK2982772 trough concentrations reached steady state by Day 2. Administration of GSK2982772 with food (standard or high-fat meal) had no apparent effect on the $C_{\max }$ or AUC compared with the fasted state (Table 3).

\subsubsection{Japanese Subjects}

In Study 2, the shape of the GSK2982772 plasma concentration-time profiles in Japanese subjects following single-day administration of $120 \mathrm{mg}$ TID and $240 \mathrm{mg}$ TID was generally similar to Western subjects except that a lower $C_{\max }$ and prolonged $T_{\max }$ for the third dose was observed in Western compared with Japanese subjects (Fig. 4). The ratios of the geometric means of $C_{\max }$ and AUC values from the first and second dose of GSK2982772 for Japanese:Western subjects were close to unity (mean ratios between 0.9 to 1.3 ) (Table 4) indicating that these pharmacokinetic parameters were similar between the two ethnic groups. The median $T_{\max }$ after the first and second oral dose of $120 \mathrm{mg}$ and $240 \mathrm{mg}$ were also similar (Fig. 5; Table 4). However, following the third dose, the geometric mean $C_{\max }$ and $\mathrm{AUC}_{(14-24)}$ were approximately 1.4-1.7 times higher for Japanese subjects than for Western subjects (Table 4). $\mathrm{AUC}_{(0-24)}$ values were similar in Western and Japanese subjects at the same dose levels (120 mg TID and $240 \mathrm{mg}$ TID). The inter-individual variability observed for $C_{\max }$ and AUC in Japanese subjects from Study 2 was less than that of Western subjects in Study 1 (Tables 2, 4). In Japanese subjects, the geometric mean $t_{1 / 2}$, determined after the third dose of the TID regimens, was similar for all three doses (5.9-7.2 h).

\section{4 $4 \beta$-Hydroxycholesterol Biomarker}

The mean (SD) plasma $4 \beta$-hydroxycholesterol-tocholesterol ratios on Day 1 and Day 15 were similar (Day 15 4 $\beta$-hydroxycholesterol/cholesterol: Day 1 $4 \beta$-hydroxycholesterol/cholesterol) [120 mg TID ( $n=10)$, $1.2(0.2) ; 240 \mathrm{mg}$ TID $(n=9), 1.1(0.3)]$ indicating that 14 days of GSK2982772 administration does not substantially change CYP3A4 activity.

\section{Discussion}

In these studies, the safety, tolerability, and pharmacokinetics of GSK2982772 were evaluated at higher doses (up to $720 \mathrm{mg} /$ day) than investigated previously (up to $240 \mathrm{mg} /$ day). 


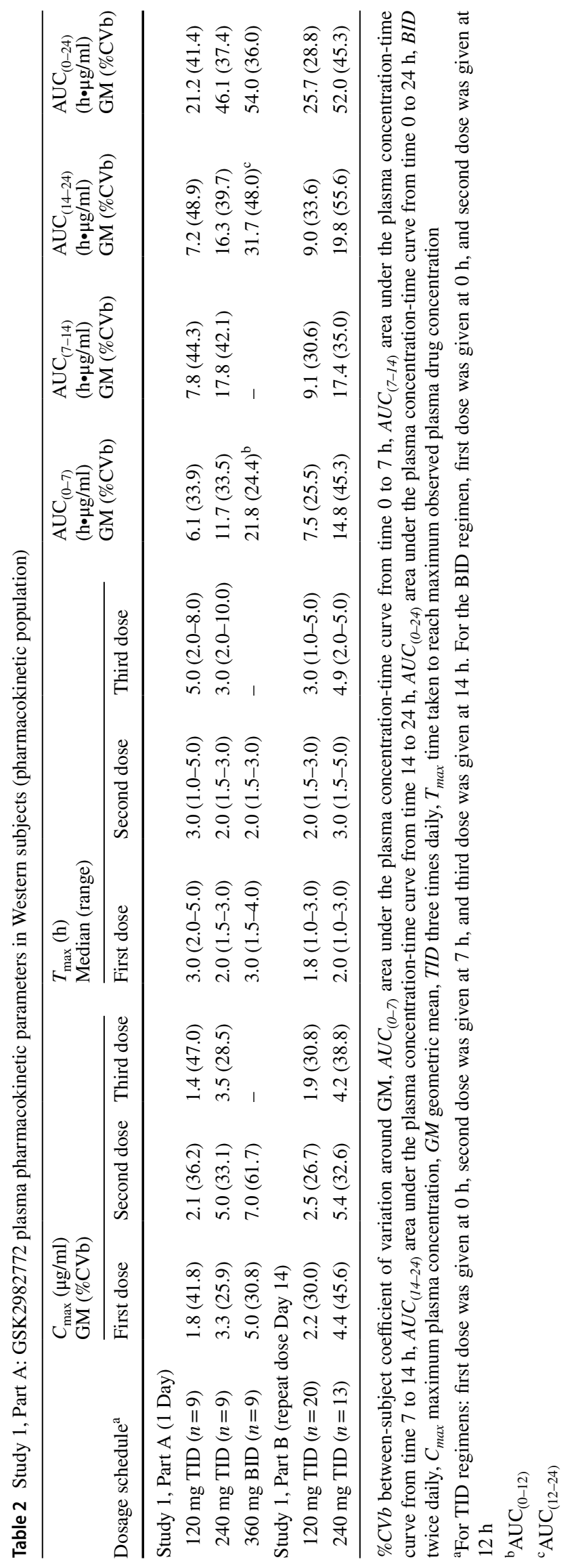


Table 3 Study 1, Part B: GSK2982772 plasma pharmacokinetic parameters in Western subjects (pharmacokinetic population)

\begin{tabular}{|c|c|c|c|c|c|c|}
\hline \multirow[b]{2}{*}{ Dosage schedule } & \multicolumn{3}{|c|}{$\begin{array}{l}C_{\max } \text { First dose }(\mu \mathrm{g} / \mathrm{ml}) \\
\mathrm{GM}(\% \mathrm{CVb})\end{array}$} & \multicolumn{3}{|c|}{$\begin{array}{l}\operatorname{AUC}_{(0-7)}(\mathrm{h} \bullet \mu \mathrm{g} / \mathrm{ml}) \\
\operatorname{GM}(\% \mathrm{CVb})\end{array}$} \\
\hline & Fasted & Standard meal & High-fat meal & Fasted & Standard meal & High-fat meal \\
\hline \multicolumn{7}{|c|}{ Study 1, Part B food effects } \\
\hline $120 \mathrm{mg}$ TID $(n=20)$ & $2.2(30.0)$ & $2.1(21.7)$ & $1.8(29.8)$ & $7.5(25.5)$ & 7.7 (31.5) & $6.9(24.6)$ \\
\hline 240 mg TID $(n=13)$ & $4.4(45.6)$ & $3.9(33.8)$ & $4.1(58.4)$ & $14.8(45.3)$ & $14.7(37.6)$ & $16.0(42.9)$ \\
\hline
\end{tabular}

$\% C V b$ between-subject coefficient of variation around $\mathrm{GM}, A U C_{(0-7)}$ area under the plasma concentration-time curve from time 0 to $7 \mathrm{~h}, C_{m a x}$ maximum plasma concentration, GM geometric mean, TID three times daily

\section{A $120 \mathrm{mg}$ TID}

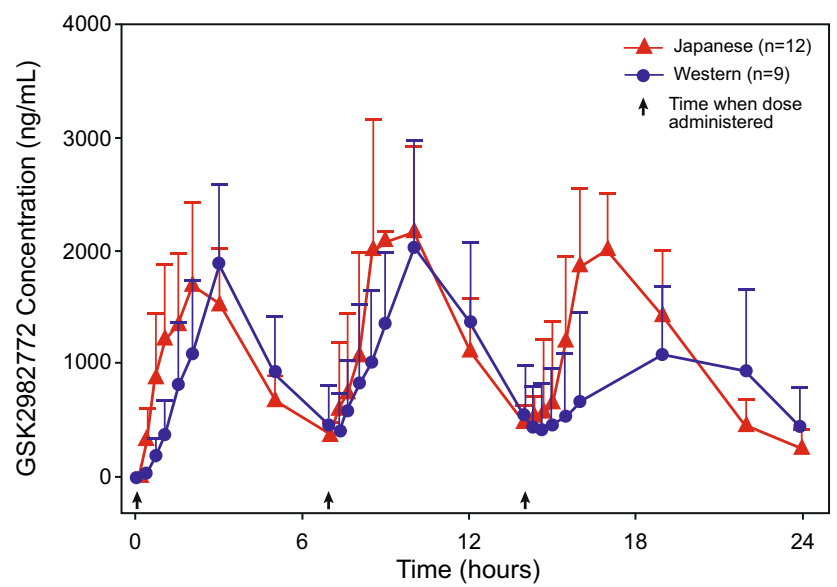

B $240 \mathrm{mg}$ TID

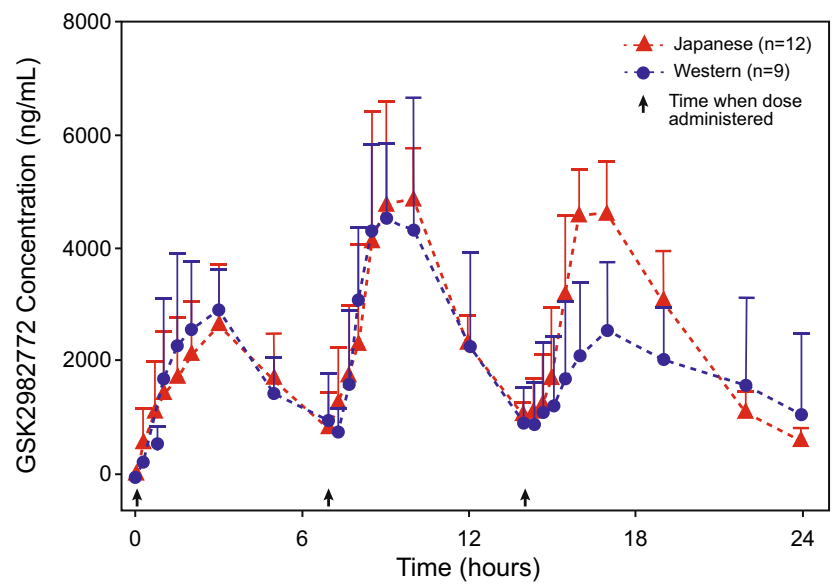

Fig. 4 Plasma GSK2982772 concentration-time profiles (arithmetic mean \pm SD) in healthy Western and Japanese subjects. a $120 \mathrm{mg}$ TID. b $240 \mathrm{mg}$ TID. $S D$ standard deviation, TID three times daily

The GSK2982772 pharmacokinetic characteristics of rapid absorption and rapid elimination were as anticipated based on the pharmacokinetics previously observed [1]. Similar to prior studies at lower dosages, the pharmacokinetics of GSK2982772 was approximately dose proportional over the dose range studied (120-240 mg TID), indicating no solubility rate-limited absorption or saturation of clearance mechanisms. Additionally, administration of GSK2982772 with food (standard or high-fat meal) had no apparent impact on pharmacokinetics compared with the fasted state, which is similar to previously reported studies showing only a slight delay in the rate of absorption under fed conditions. There was no apparent accumulation of GSK2982772 following repeat BID or TID dosing, and steady state was reached by Day 2 of repeat dosing.

For the graphical comparisons of GSK2982772 pharmacokinetics, the mean concentration-time profiles of Japanese subjects following the first and second dose were almost superimposable and AUC and $C_{\max }$ values (120 and $240 \mathrm{mg}$ TID) were generally consistent with values in Western subjects. This observation was further supported by post hoc analyses of the ratios of the geometric means of $C_{\max }$ and AUC values between Japanese and Western subjects, where the ratios generated from the first two doses were close to unity.

However, following administration of the third dose, the rate of absorption was slower in Western subjects compared with Japanese subjects. Differences in absorption may be explained by the posture of subjects around the administration time of the third dose. Japanese subjects were maintained in a seated or semi-seated position for $2 \mathrm{~h}$ following the third dose. While a specific posture was not stipulated for Western subjects, the third dose was administered between 9:00 p.m. and 11:15 p.m., which coincided with bedtime. Adopting the recumbent position, which is known to slow gastric emptying [9], was more likely in Western subjects and could contribute to the later $T_{\max }$ and lower $C_{\max }$ observed after the third versus the second dose.

The $t_{1 / 2}$ observed in Japanese subjects was consistent with that observed following a single oral dose in Western subjects [1].

Currently, an important pathway of GSK2982772 elimination is considered to be glucuronidation catalysed by UGT1A9. Variants of UGT1A9 [10] can contribute to variation in pharmacokinetics of UGT1A9 substrates, but an association is not always observed [11-17]. While the 


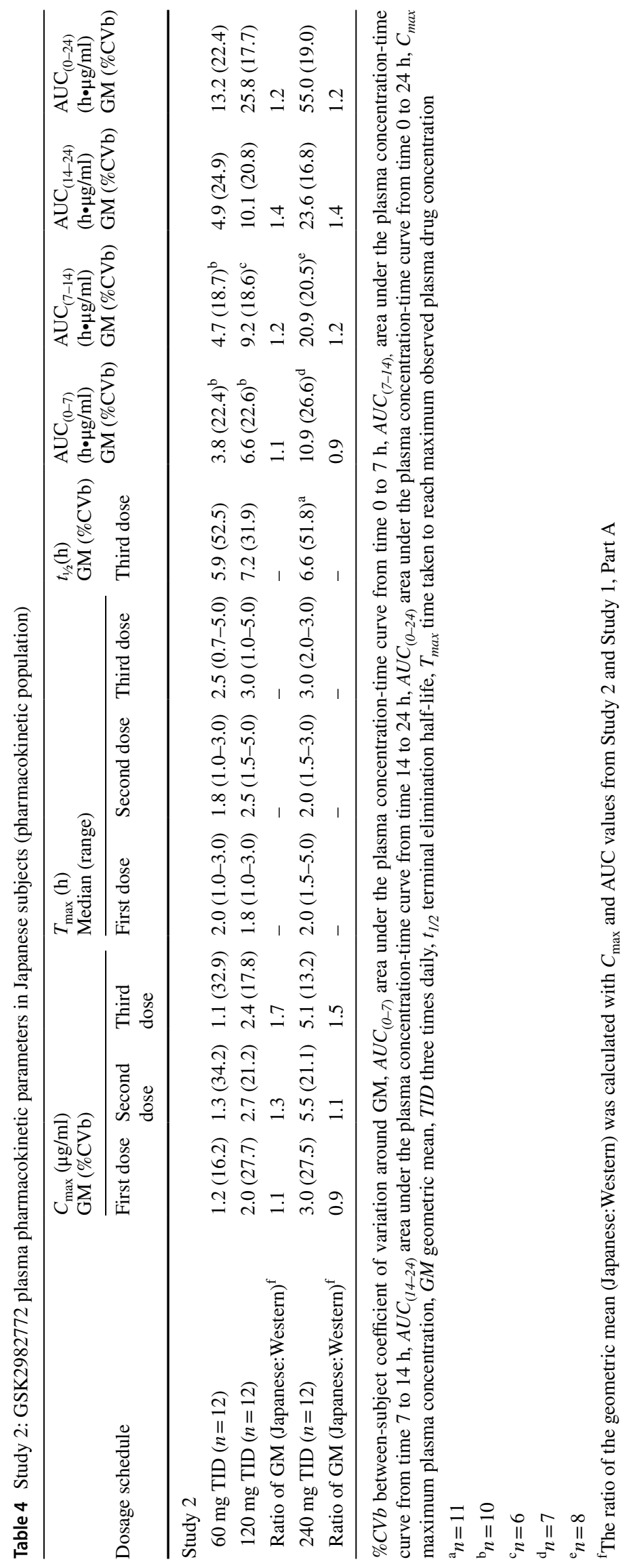




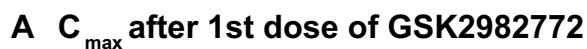

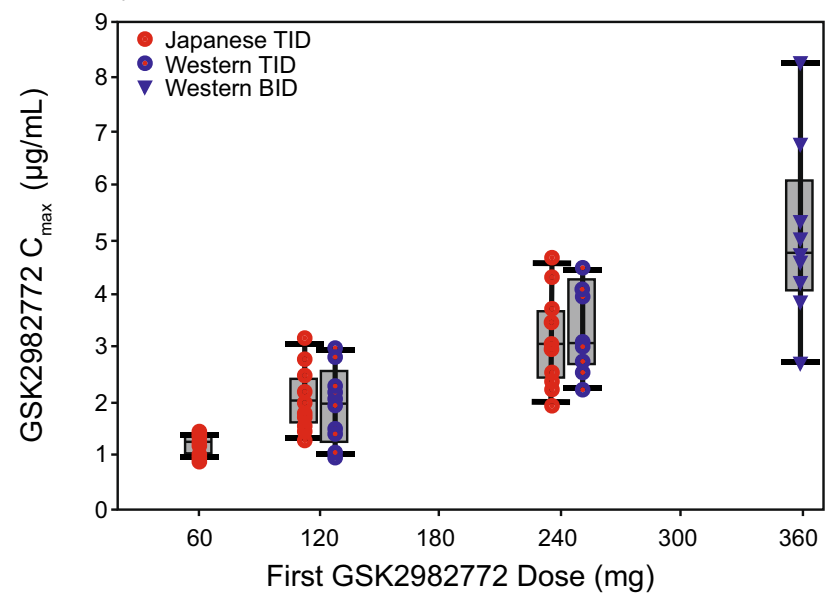

B AUC $_{(0-7)}$ after 1st dose of GSK2982772
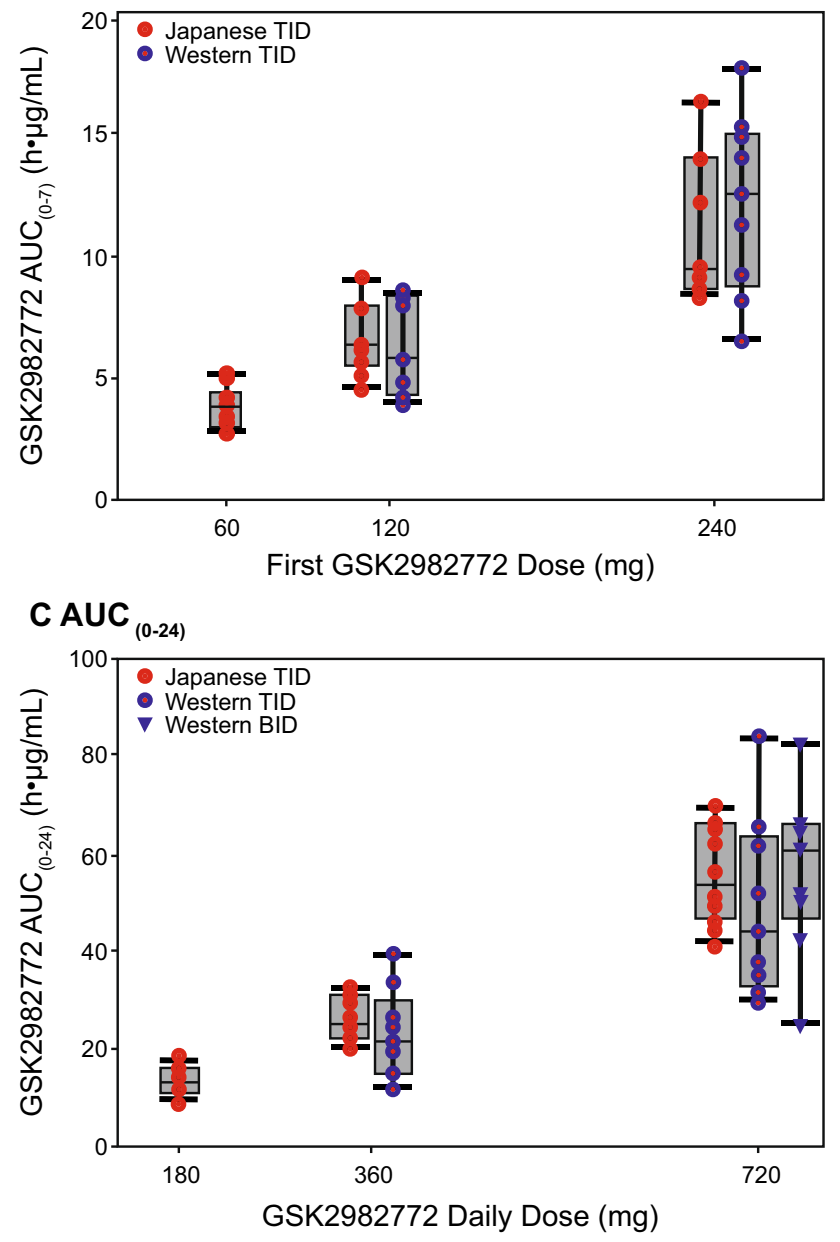

Fig. 5 Comparison of GSK2982772 $C_{\text {max }}, \mathrm{AUC}_{(0-7)}$, and $\mathrm{AUC}_{(0-24)}$ in healthy Western and Japanese subjects. a $C_{\max }$ after first dose of GSK2982772. b $A C_{(0-7)}$ after first dose of GSK2982772. c AUC $(0-24)$. Boxes represent the 25 th-75th percentiles with median shown and whiskers represent the 10th-90th percentiles with individual data points superimposed. $A U C_{(0-7)}$ area under the plasma concentrationtime curve from time 0 to $7 \mathrm{~h}, A U C_{(0-24)}$ area under the plasma concentration-time curve from time zero to $24 \mathrm{~h}, B I D$ twice daily, $C_{\max }$ maximum plasma concentration, TID three times daily frequencies of UGT1A9 variants with functional consequences can differ between East Asian and European populations [10], for GSK2982772, the pharmacokinetics were similar in the healthy subjects of Japanese and European ancestry studied.

There was no increase detected in the mean plasma $4 \beta$-hydroxycholesterol-to-cholesterol ratio following 14 days of repeat dose administration, suggesting that GSK2982772 is not a potent inducer of CYP3A4 up to doses of $240 \mathrm{mg}$ TID.

GSK2982772 was well tolerated in healthy subjects. The majority of AEs in both studies were mild in intensity. Contact dermatitis was the most common AE, consistent with previous findings at lower doses of GSK2982772 [1]. In Study 1, there was one severe, but non-serious, asymptomatic study drug-related event of transaminase elevation, which resolved after drug discontinuation. No further events occurred in higher dose cohorts. Although an increase in NSVT events was observed in Study 1, several factors suggest they were unlikely to be related to study medication. The events occurred at a time beyond elimination of the compound (i.e., 5 half-lives) in three of four subjects. Furthermore, no significant proportional differences in the occurrence rates between active drug and placebo were noted, and the incidence of these rhythm variants was not different from what is expected in similar healthy populations $[18,19]$. The arrhythmias observed in the healthy subjects appeared to be heterogeneous and of diverse mechanisms. Some were automatic, some may have been reentrant, and none were polymorphous ventricular tachycardia (PMVT). All cases appeared to be monomorphic VT. No QT prolongation has been seen in the development program to date, and the NSVT events had no corresponding QT prolongation. Likewise, there were no associated ST segment changes or QRS prolongation. It is unlikely that a single, underlying, pro-arrhythmic mechanism with GSK2982772 exposure exists.

\section{Conclusions}

The results from the two studies demonstrate that GSK2982772 is well tolerated in healthy Japanese and Western subjects up to a daily dose of $720 \mathrm{mg}$. The pharmacokinetics of GSK2982772 was generally similar between Japanese and Western subjects, with differences in parameters being partly explained by study differences in dose administration procedures. Similar safety and pharmacokinetics results from these studies support participation of Japanese subjects in future global phase studies and the examination of GSK2982772 (up to $720 \mathrm{mg}$ daily) as a potential therapy for treatment of IMIDs. 
Acknowledgements Funding for the studies (NCT03305419 and NCT03590613 available from http://www.clinicaltrials.gov) was provided by GlaxoSmithKline (GSK). All listed authors meet the criteria for authorship set forth by the International Committee for Medical Journal Editors. Editorial support (assembling tables and figures, collating author comments, copyediting, fact checking, and referencing) (Allyson Lehrman, DPM) and graphic services were provided by AOIC, LLC, and were funded by GSK. The authors also thank Farjana Mahammed of GSK for her confirmation of the bioanalytical method validation for the Western study.

\section{Declarations}

Funding Funding for the studies (NCT03305419 and NCT03590613 available from http://www.clinicaltrials.gov) was provided by GlaxoSmithKline (GSK).

Conflict of interest Debra J. Tompson, Carwyn Davies, Nicola E. Scott, Edward P. Cannons, Annette S. Gross, Marcy Powell, Ryutaro Shimamura, Takashi Nagakubo, Atsushi Nakano are employees of and hold equity stock in GlaxoSmithKline. Hirofumi Ogura and Harue Igarashi are employees of GSK. Michalis Kostapanos is an NHS consultant working with a 50\% secondment in GSK's Clinical Unit Cambridge and has no other relevant conflicts of interests for this study. Hiroko Ino is a former employee of GSK.

Ethics approval For each study, approval was from the investigational centre ethics committee or institutional review board (Study 1: NRES Committee East of England, Cambridgeshire and Hertfordshire, Rolling Mill Road, Jarrow, Tyne and Wear, UK, NE32 3DT; Study 2: Hakata Clinic Institutional Review Board, Random Square 5-7 FL, 6-18 Tenyamachi, Hakata-ku, Fukuoka Japan, 812-0025). The studies were conducted in accordance with the International Conference on Harmonisation of Technical Requirements for Registration of Pharmaceuticals for Human Use, Good Clinical Practice and applicable country-specific requirements and the ethical principles that are outlined in the Declaration of Helsinki.

Consent to participate Written informed consent was obtained from each subject prior to any study-related activities.

Availability of data and material Within 6 months of this publication, anonymised individual subject data, the annotated case report forms, protocols, reporting and analysis plans, data set specifications, raw datasets, analysis-ready datasets, and clinical study reports will be available for research proposals approved by an independent review committee. Proposals should be submitted to http://www.clinicalst udydatarequest.com. A data access agreement will be required.

Code availability Statistical analyses were performed with SAS (version 9.2 or higher; Cary, NC, USA).

Author contributions Debra J. Tompson and Marcy Powell: contributed to the conception or design of the high-dose pharmacokinetic study; Debra J. Tompson, Hiroko Ino, Takashi Nagakubo, Atsushi Nakano, Ryutaro Shimamura contributed to the conception or design of the Japanese study. Debra J. Tompson, Edward P. Cannons, Michalis Kostapanos, Marcy Powell, Hiroko Ino, Hirofumi Ogura, and Harue Igarashi contributed to the acquisition of the data; Debra J. Tompson, Nicola E. Scott, Carwyn Davies, Annette S. Gross, Marcy Powell, Hiroko Ino, Ryutaro Shimamura, Hirofumi Ogura, Takashi Nagakubo, Harue Igarashi, and Atsushi Nakano contributed to the data analysis or interpretation. All authors provided critical review, final approval of the publication, and agree to be accountable for the contents of this work.
Open Access This article is licensed under a Creative Commons Attribution-NonCommercial 4.0 International License, which permits any non-commercial use, sharing, adaptation, distribution and reproduction in any medium or format, as long as you give appropriate credit to the original author(s) and the source, provide a link to the Creative Commons licence, and indicate if changes were made. The images or other third party material in this article are included in the article's Creative Commons licence, unless indicated otherwise in a credit line to the material. If material is not included in the article's Creative Commons licence and your intended use is not permitted by statutory regulation or exceeds the permitted use, you will need to obtain permission directly from the copyright holder. To view a copy of this licence, visit http://creativecommons.org/licenses/by-nc/4.0/.

\section{References}

1. Weisel K, Scott NE, Tompson DJ, Votta BJ, Madhavan S, Povey $\mathrm{K}$, et al. Randomized clinical study of safety, pharmacokinetics, and pharmacodynamics of RIPK1 inhibitor GSK2982772 in healthy volunteers. Pharmacol Res Perspect. 2017. https:// doi.org/10.1002/prp2.365.

2. Zhou W, Yuan J. Necroptosis in health and diseases. Semin Cell Dev Biol. 2014;35:14-23.

3. Kopalli SR, Kang T-B, Koppula S. Necroptosis inhibitors as therapeutic targets in inflammation mediated disorders-a review of the current literature and patents. Expert Opin Ther Patents. 2016;26(11):1239-56.

4. Weisel K, Berger S, Papp K, Maari C, Krueger J, et al. Response to inhibition of receptor-interacting protein kinase 1 (RIPK1) in active psoriasis: a randomized placebo-controlled study. J Clin Pharmacol Ther; 2020. https://doi.org/10.1002/cpt.1852.

5. Mao J, Martin I, McLeod J, Nolan G, van Horn R, et al. Perspective: 4beta-hydroxycholesterol as an emerging endogenous biomarker of hepatic CYP3A. Drug Metab Rev. 2017;49(1):18-34.

6. Assessing the effects of food on drugs in INDs and NDAsclinical pharmacology considerations: guidance for industry. In: US Department of Health and Human Services. Accessed 3 Apr 2019.

7. Bioanalytical method validation — guidance for industry. In: US Department of Health and Human Services. Accessed 18 Aug 2020.

8. Guideline on bioanalytical method validation. In: European Medicines Agency. Accessed 18 Aug 2020.

9. Queckenberg C, Fuhr U. Influence of posture on pharmacokinetics. Eur J Clin Pharmacol. 2009;65(2):109-19.

10. Maeda H, Hazama S, Shavkat A, Okamoto K, Oba K, Sakamoto J, et al. Differences in UGT1A1, UGT1A7, and UGT1A9 polymorphisms between Uzbek and Japanese populations. Mol Diagn Ther. 2014;18(3):333-42.

11. Xie XC, Li J, Wang HY, Li HL, Liu J, Fu Q, et al. Associations of UDP-glucuronosyltransferases polymorphisms with mycophenolate mofetil pharmacokinetics in Chinese renal transplant patients. Acta Pharmacol Sin. 2015;36(5):644-50.

12. Mazidi T, Rouini, Ghahremani MH, Dashti-Khavidaki S, LessanPezeshki M, Ahmadi FL, et al. Impact of UGT1A9 polymorphism on mycophenolic acid pharmacokinetic parameters in stable renal transplant patients. Iran J Pharm Res. 2013;12(3):547-56.

13. Inoue K, Miura M, Satoh S, Kagaya H, Saito M, Habuchi T, et al. Influence of UGT1A7 and UGT1A9 intronic I399 genetic polymorphisms on mycophenolic acid pharmacokinetics in Japanese renal transplant recipients. Ther Drug Monit. 2007;29(3):299-304.

14. Sandanaraj E, Jada SR, Shu X, Lim R, Lee SC, Zhou Q, et al. Influence of UGT1A9 intronic I399C $>$ T polymorphism on 
SN-38 glucuronidation in Asian cancer patients. Pharmacogenomics J. 2008;8(3):174-85.

15. Han JY, Lim HS, Park YH, Lee SY, Lee JS. Integrated pharmacogenetic prediction of irinotecan pharmacokinetics and toxicity in patients with advanced non-small cell lung cancer. Lung Cancer (Amsterdam, Netherlands). 2009;63(1):115-20.

16. Cecchin E, Innocenti F, D'Andrea M, Corona G, De Mattia E, Biason P, et al. Predictive role of the UGT1A1, UGT1A7, and UGT1A9 genetic variants and their haplotypes on the outcome of metastatic colorectal cancer patients treated with fluorouracil, leucovorin, and irinotecan. J Clin Oncol. 2009;27(15):2457-65.

17. van Schaik RH, van Agteren M, de Fijter JW, Hartmann A, Schmidt J, Budde K, et al. UGT1A9 -275T > A/- 2152C $>$ T polymorphisms correlate with low MPA exposure and acute rejection in $\mathrm{MMF} /$ tacrolimus-treated kidney transplant patients. Clin Pharmacol Ther. 2009;86(3):319-27.

18. Hingorani P, Karnad, Rohekar B, Kerkar V, Lokhandwala YY, Kothari S. Arrhythmias seen in baseline 24-hour Holter ECG recordings in healthy normal volunteers during phase 1 clinical trials. J Clin Pharmacol. 2016;56(7):885-93.

19. Min SS, Turner JR, Nada A, DiMino TL, Hynie I, Kleiman R, et al. Evaluation of ventricular arrhythmias in early clinical pharmacology trials and potential consequences for later development. Am Heart J. 2010;159(5):716-29.

\section{Affiliations}

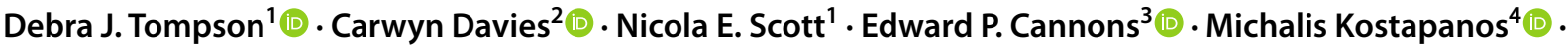

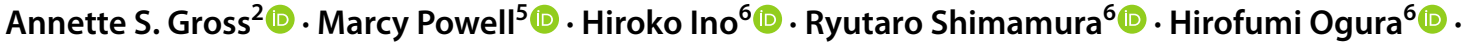

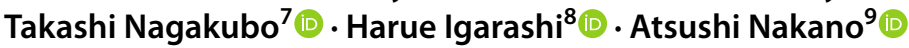

1 Clinical Pharmacology Modelling and Simulation, GlaxoSmithKline, Medicines Research Centre, Gunnels Wood Road, Stevenage, Hertfordshire, UK

2 Clinical Pharmacology Modelling and Simulation, GlaxoSmithKline, Sydney, NSW, Australia

3 Global Clinical Sciences and Delivery, GlaxoSmithKline, Medicines Research Centre, Stevenage, Hertfordshire, UK

4 Clinical Care Unit Cambridge, GlaxoSmithKline, and Addenbrooke's Hospital, Cambridge University Hospitals NHS Foundation Trust, Cambridge, Cambridgeshire, UK
5 Safety and Medical Governance, GlaxoSmithKline, Research Triangle Park, NC, USA

6 Clinical Pharmacology, Medicines Development, GlaxoSmithKline, Tokyo, Japan

7 Biomedical Data Sciences Department, GlaxoSmithKline, Tokyo, Japan

8 Pre-Clinical Development Department, GlaxoSmithKline, Tokyo, Japan

9 Medicines Development, GlaxoSmithKline, Tokyo, Japan 